\title{
SEARCH FOR DEEPLY BOUND KAONIC NUCLEAR STATES IN THE AMADEUS EXPERIMENT*
}

\author{
Magdalena SkurzoK $^{\mathrm{a}}$, Michael Cargnelli ${ }^{\mathrm{b}}$ \\ Catalina Curceanu ${ }^{c}$, Raffaele Del Grande ${ }^{\mathrm{c}}$, Laura Fabbiettid ${ }^{\mathrm{d}, \mathrm{e}}$ \\ Carlo Guaraldo ${ }^{\mathrm{c}}$, Johann Marton ${ }^{\mathrm{b}}$, Pawee Moskal ${ }^{\mathrm{a}}$ \\ Kristian Piscicchia ${ }^{\mathrm{f}, \mathrm{c}}$, Alessandro $\mathrm{Scordo}^{\mathrm{c}}$, Michą Silarski $^{\mathrm{a}}$ \\ Diana Laura Sirghi ${ }^{\mathrm{c}}$, Ivana Tucakovic ${ }^{\mathrm{g}}$, Oton Vazquez Doce ${ }^{\mathrm{d}, \mathrm{e}}$ \\ SŁaWOMIR WYCECH ${ }^{\mathrm{h}}$, EBerhard Widmann ${ }^{\mathrm{b}}$, JOHANN ZMESKal ${ }^{\mathrm{b}}$ \\ ${ }^{a}$ M. Smoluchowski Institute of Physics, Jagiellonian University, Kraków, Poland \\ ${ }^{\mathrm{b}}$ Stefan-Meyer-Institut für Subatomare Physik, Vienna, Austria \\ ${ }^{\mathrm{c}}$ INFN - Laboratori Nazionali di Frascati, Frascati, Italy \\ dExcellence Cluster "Origin and Structure of the Universe" \\ 85748 Garching, Germany

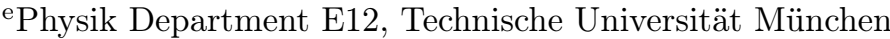 \\ 85748 Garching, Germany \\ ${ }^{\mathrm{f}}$ CENTRO FERMI - Museo Storico della Fisica e Centro Studi e Ricerche \\ "Enrico Fermi", Roma, Italy \\ ${ }^{g}$ Ruđer Bošković Institute, Zagreb, Croatia \\ ${ }^{\mathrm{h}}$ National Centre for Nuclear Research, Warszawa, Poland
}

(Received January 4, 2018)

We briefly report on the search for Deeply Bound Kaonic Nuclear States with AMADEUS in the $\Sigma^{0} p$ channel, and future perspectives.

DOI:10.5506/APhysPolB.49.705

\section{Introduction}

The existence or not of the Deeply Bound Kaonic Nuclear States (DBKNS) is currently one of the hottest topics in nuclear and hadronic strangeness physics, both from experimental and theoretical points of view. The existence of bound kaonic nuclear states of $K^{-}$, also called kaonic nuclear clusters, was firstly predicted in 1986 [1]. The position of the $\Lambda(1405)$ reflects the strength of the $\bar{K} N$ interaction, thus influencing the possible formation of $\bar{K}$ multi-nucleon bound states. Accordingly, recent theoretical calculations,

* Presented at the XXXV Mazurian Lakes Conference on Physics, Piaski, Poland, September 3-9, 2017. 
based on different approaches, deliver a wide range of bindings and widths for the di-baryonic kaonic bound state $p p K^{-}[2-7]$, while the experimental results are contradictory [8-14]. Moreover, the extraction of a $p p K^{-}$signal in $K^{-}$absorption experiments is strongly affected by the yield and the shape of the competing $\mathrm{K}^{-}$multi-nucleon absorption processes. Therefore, in order to clarify this issue, experimental data are needed. This research is very important in understanding the fundamental laws of the Nature and Universe. It can have important consequences in various sectors of physics, such as nuclear and particle physics, as well as astrophysics. The binding of the kaon in nuclear medium may have impact on models describing the structure of neutron stars (Equation of State of neutron stars) [15, 16] including binaries which are expected to be sources of the gravitational waves. Investigation of stable forms of strange matter like DBKNS in extreme conditions would be helpful for a better understanding of elementary kaon-nucleon interaction for low energies in the non-perturbative quantum chromodynamics (QCD) and would contribute to solving crucial problems in hadron physics: hadron masses (related to the chiral symmetry breaking), hadron interactions in nuclear medium and the structure of the dense nuclear matter.

The AMADEUS group has developed a method having a high chance for a discovery of DBKNS corresponding to $K^{-} p p, K^{-} p p n$ and $K^{-} p p n n$ kaonic nuclear clusters and their decays to $\Sigma^{0} / \Lambda p, \Sigma^{0} / \Lambda d$ and $\Sigma^{0} / \Lambda t$, respectively. The method is based on the exclusive measurement of the momentum, angular and invariant mass spectra for correlated $\Sigma^{0} / \Lambda p, \Sigma^{0} / \Lambda d$, $\Sigma^{0} / \Lambda t$ pairs [17]. Possible DBKNS may be produced with $K^{-}$stopped in helium or carbon and then decaying into considered decay channels. The AMADEUS experiment is conceived to perform a systematic investigation of the low-energy $\bar{K} N$ interaction, taking advantage of the kaon beams delivered by the DA $\Phi N E$ collider [18]. The ongoing AMADEUS analyses refer to two data samples. One is represented by the data collected by the KLOE Collaboration [19] during the 2004/2005 data taking, corresponding to $\sim 1.74 \mathrm{fb}^{-1}$. The KLOE detector [20] is used as an active target, the hadronic interaction of negative kaons with the materials of the apparatus being investigated. This includes, in particular, $K^{-9} \mathrm{Be}$ absorptions in the DA $\Phi N E$ thin beryllium cylindrical layer and the DA $\Phi$ NE aluminated beryllium pipe, $K^{-12} \mathrm{C}$ and $K^{-} \mathrm{H}$ absorptions in the KLOE Drift Chamber [21] (DC) inner wall (aluminated carbon fiber), and $K^{-4} \mathrm{He}$ in the DC gas. Extremely rich experimental information is contained in this sample, with $K^{-}$hadronic captures both at rest and in flight [22]. In order to increase the statistics, a high purity carbon target (graphite) was realized in the summer of 2012 and installed inside the KLOE detector, between the beam pipe and the DC inner wall. This setup is crucial because it serves as an essential interpretation tool. The geometry of the target was optimized to maximize the kaon stopping power. The total collected integrated 
luminosity is $\sim 90 \mathrm{pb}^{-1}$. Up to now, we analysed a sample of $37 \mathrm{pb}^{-1}$ reconstructed data. The adopted procedure is to exclusively measure the correlated $\Sigma^{0} / \Lambda p, \Sigma^{0} / \Lambda d$ and $\Sigma^{0} / \Lambda t$ pairs, searching for the production of $K^{-} p p, K^{-} p p n$ and $K^{-}$ppnn multi-nucleon, and related bound states. In the next section, we briefly present the results of data analysis devoted to the $\Sigma^{0} p$ channel. A detailed description can be found in Ref. [23].

\section{2. $\Sigma^{0} p$ data analysis}

The analysis presented below is focused on studies of $K^{-}$absorption processes inside the DC carbon wall leading to the $\Sigma^{0} p$ final state [23]. Selection of $\Lambda(1116)$ hyperons, being the signature of the $K^{-}$hadronic interaction in ${ }^{12} \mathrm{C}$, was the first aim of the data analysis. $\Lambda$ s were identified via reconstruction of their decay vertex $\Lambda \rightarrow p+\pi^{-}(\mathrm{BR}=63.8 \%)$. The selection and identification of protons and negatively charged pions was done using the $\mathrm{d} E / \mathrm{d} x$ information in the DC installed around the interaction point and the momentum of the track. The proton-pion invariant mass was determined under the $p$ and $\pi^{-}$mass hypothesis. The constructed $p \pi^{-}$invariant mass resulted in a value of $1115.753 \pm 0.002 \mathrm{MeV} / c^{2}$ and a statistical error of $0.5 \mathrm{MeV} / c^{2}$. Then, the $\Lambda$-proton vertex was searched for, and $K^{-}$absorption processes inside the DC wall were selected based on the radial position $\rho_{\Lambda p}$. The identification of $\Sigma^{0}$ candidates was carried out through their decay into $\Lambda \gamma$ pairs, where $\gamma$ quanta were identified in calorimeter [24]. Afterwards, a simultaneous global fit to the $\Sigma^{0} p$ invariant mass, the $\cos \left(\theta_{\Sigma^{0} p}\right)$, the $\Sigma^{0}$ and the proton momenta was performed in order to extract contributions from the different absorption processes like 2-Nucleon Absorption (2NA) with final-state interaction (FSI) or without it $(\mathrm{QF}), 3 \mathrm{NA}$ and $4 \mathrm{NA}$. For this purpose, Monte Carlo simulations of the absorption processes in ${ }^{12} \mathrm{C}$ were carried out. The final fit is presented in Figs. 1 and 2. It determined the contribution of the different absorption processes. The fit results in the first measurement of the 2NA free from FSI, which was found to be $12 \%$ of the total absorption cross section. Contribution from the $K^{-} p p$ bound state was included in the second fit of the experimental data. A Breit-Wigner shape was used to simulate the bound state contribution, scanning a grid of possible bindings $\left(15-75 \mathrm{MeV} / c^{2}\right.$ in steps of $\left.15 \mathrm{MeV} / c^{2}\right)$ and widths $(30$ $70 \mathrm{MeV} / c^{2}$ in steps of $\left.20 \mathrm{MeV} / c^{2}\right)$. The best value of the total reduced $\chi^{2}$ was achieved for the hypothesis of a binding energy of $45 \mathrm{MeV} / c^{2}$ and width $30 \mathrm{MeV} / c^{2}$, as shown in Fig. 3. The $K^{-} p p$ yield extracted from the fit is $K^{-} p p / K_{\text {stop }}^{-}=\left(0.044 \pm 0.009\right.$ stat. $_{-0.005}^{+0.004}$ syst. $) \times 10^{-2}$. However, an F-Test was carried out to compare the two models (with and without a $K^{-} p p$ bound state) results in a $1 \sigma$ significance for the best fit which does not allow to claim the observation of a bound state. 

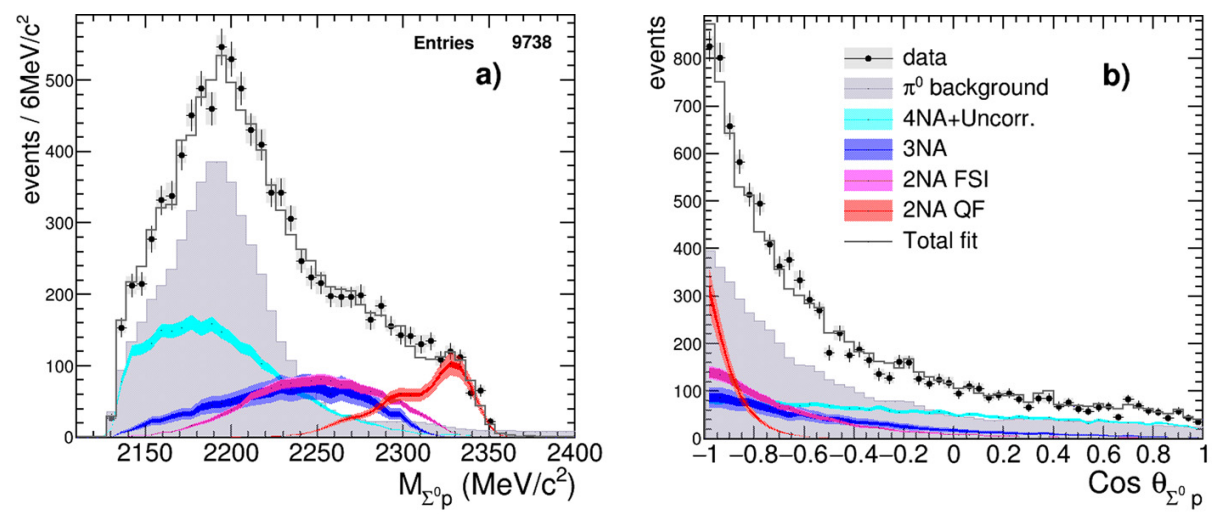

Fig. 1. (Colour on-line) Simultaneous fit to the $\Sigma^{0} p$ invariant mass (a), $\cos \left(\Sigma^{0} p\right)$ (b), $\Sigma^{0}$ momentum and proton momentum distributions (see Fig. 2) performed for kaons stopped in the DC wall. The colors represent the following fit contributions: $\pi^{0}$ background (grey), 4NA with uncorrelated production of the $\Sigma^{0} p$ final state (cyan), 3NA (blue), 2NA-FSI (magenta) and 2NA-QF (red). The lightcoloured bands correspond to the fit errors, while the darker bands represent the symmetrized systematic errors. The grey line shows the overall fit. Figure adopted from Ref. [23].
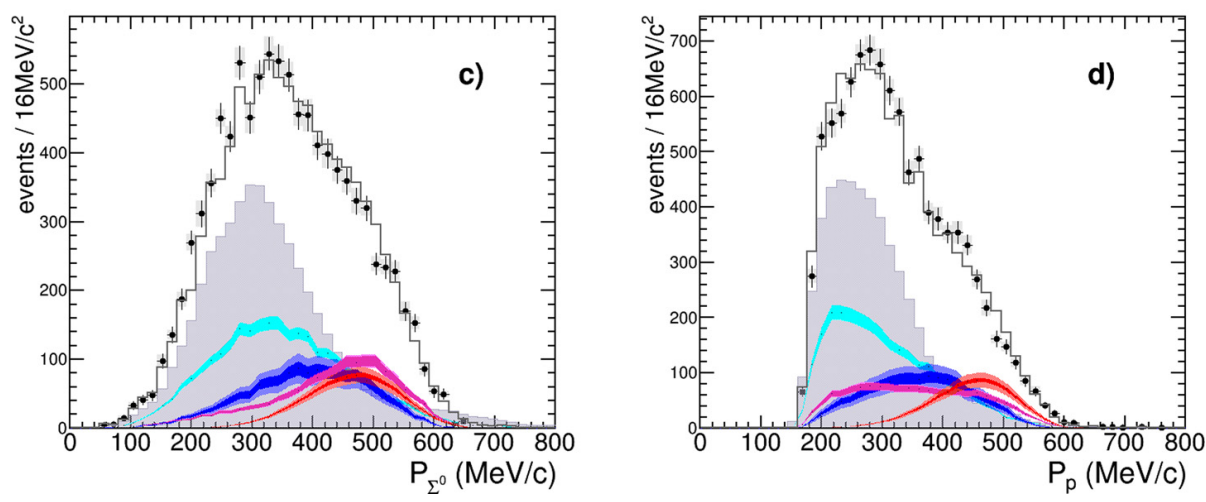

Fig. 2. (Colour on-line) The same as Fig. 1 for $\Sigma^{0}$ momentum (c) and proton momentum distributions (d). Figure adopted from Ref. [23]. 

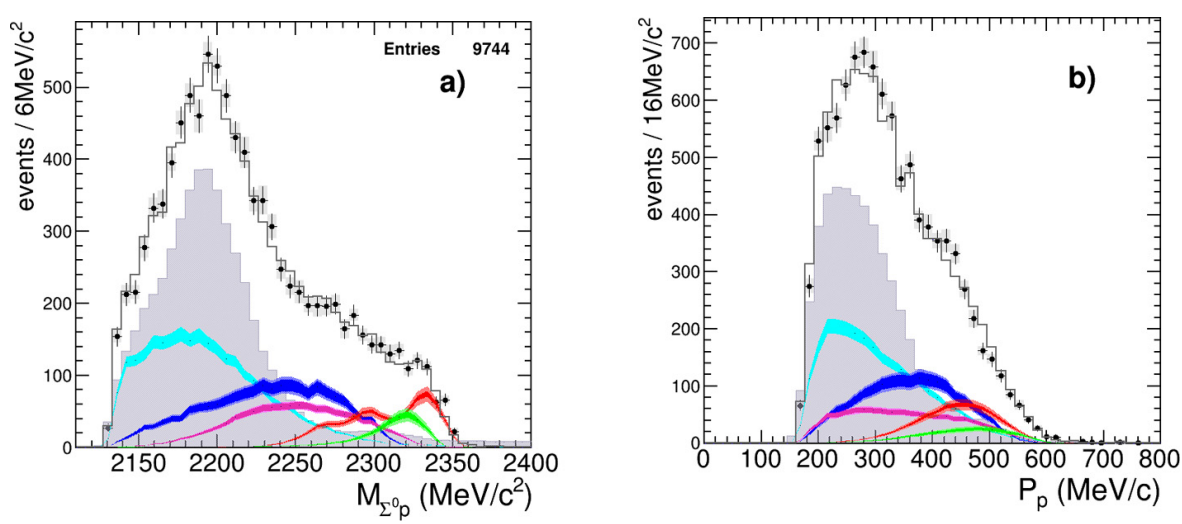

Fig. 3. (Colour on-line) Simultaneous fit to the $\Sigma^{0} p$ invariant mass (a) and proton momentum distributions (b) performed for kaons stopped in the DC wall. Bottom (green) line represents the $K^{-} p p$ signal while different contributions are marked as in Fig. 1. The black line shows the overall fit. Figure adopted from Ref. [23].

\section{Summary and conclusion}

One of the AMADEUS's goals is to search for Deeply Bound Kaonic Nuclear States by studies of $K^{-}$absorption processes in various tergets. In this work, the investigation of $\Sigma^{0} p$ final state was presented. It results in the extraction of contributions from various few-nucleon absorption processes. Contribution from a possible $K^{-} p p$ bound state was determined, however, the significance of the result is not sufficient to claim the observation of a bound system. Presently, the analysis is in progress for other channels such as $\Lambda p, \Lambda d$ and $\Lambda t$. Moreover, a feasibility study [25, 26] is ongoing for the realization of a dedicated AMADEUS experimental setup, in order to deepen and extend the low-energy anti-kaon nuclei interaction studies and obtain a fundamental input for the understanding of the QCD with strangeness.

We acknowledge the KLOE Collaboration for their support and for having provided us with the data and the tools to perform the analysis presented in this paper. We thank as well the DA $\Phi$ NE staff for the excellent working conditions and permanent support. We acknowledge the CENTRO FERMI - Museo Storico della Fisica e Centro Studi e Ricerche "Enrico Fermi" for the project PAMQ. Part of this work was supported by the Austrian Science Fund (FWF): [P24756-N20]; Austrian Federal Ministry of Science and Research BMBWK 650962/0001 VI/2/2009; the Grantïn-Aid for Specially Promoted Research (20002003); Minstero degli Affari Esteri e della Cooperazione Internazionale, Direzione Generale per la Promozione del Sistema Paese (MAECI), Strange Matter project; National Science Centre, Poland (NCN) through grant No. UMO-2016/21/D/ST2/01155. 


\section{REFERENCES}

[1] S. Wycech, Nucl. Phys. A 450, 399c (1986).

[2] T. Yamazaki et al., Phys. Rev. C 76, 045201 (2007).

[3] S. Wycech et al., Phys. Rev. C 79, 014001 (2009).

[4] N. Barnea et al., Phys. Lett. B 712, 132 (2012).

[5] E. Oset et al., Nucl. Phys. A 881, 127 (2012).

[6] A. Dote, T. Hyodo, W. Weise, Phys. Rev. C 79, 014003 (2009).

[7] Y. Ikeda, T. Sato, Phys. Rev. C 79, 035201 (2009).

[8] G. Agakishiev et al. [HADES Collaboration], Phys. Lett. B 742, 242 (2015).

[9] M. Agnello et al. [FINUDA Collaboration], Phys. Rev. Lett. 94, 212303 (2005).

[10] T. Yamazaki et al., Phys. Rev. Lett. 104, 132502 (2010).

[11] Y. Ichikawa et al., Prog. Theor. Exp. Phys. 2015, 021D01 (2015).

[12] A.O. Tokiyasu et al., Phys. Lett. B 728, 616 (2014).

[13] L. Fabbietti et al., Nucl. Phys. A 914, 60 (2013).

[14] T. Hashimoto et al., Prog. Theor. Exp. Phys. 2015, 061D01 (2015).

[15] A.E. Nelson, D.B. Kaplan, Phys. Lett. B 192, 193 (1987).

[16] A. Scordo et al., AIP Conf. Proc. 1735, 080015 (2016).

[17] C. Curceanu et al., Acta Phys. Pol. B 46, 203 (2015).

[18] G.V. Vignola et al., Conf. Proc. C 930517, 1993 (1993).

[19] F. Bossi et al. [KLOE Collaboration], Riv. Nuovo Cim. 31, 531 (2008).

[20] F. Ambrosino et al., Nucl. Instrum. Methods Phys. Res. A 534, 403 (2004).

[21] M. Adinolfi et al. [KLOE Collaboration], Nucl. Instrum. Methods Phys. Res. A 488, 51 (2002).

[22] K. Piscicchia et al., PoS Bormio2013, 034 (2013).

[23] O. Vazques Doce et al., Phys. Lett. B 758, 134 (2016).

[24] M. Adinolfi et al. [KLOE Collaboration], Nucl. Instum. Methods Phys. Res. A 482, 364 (2002).

[25] M. Bazzi et al., Nucl. Instrum. Methods Phys. Res. A 671, 125 (2012).

[26] M. Bazzi et al., JINST 8, T05006 (2013). 